\title{
VER Y PENSAR. FISIOLOGÍA MECANICISTA CARTESIANA Y FENOMENOLOGÍA DEL CUERPO
}

\author{
Esteban Garcia \\ Universidad de Buenos Aires- Consejo Nacional de \\ Investigaciones Científicas y Técnicas \\ baneste72@gmail.com \\ Paula Castelli \\ alicamento@yahoo.com
}

UBA - Agencia Nacional para la Promoción Científica y Tecnológica

\begin{abstract}
Resumen / Abstract
Merleau-Ponty como lector tenaz y riguroso del corpus cartesiano fue especialmente sensible a sus ambigüedades. Por un lado, el intelectualismo de la teoría cartesiana de la percepción (Dioptrique) es subsidiario de una fisiología mecanicista (Traité de l'Homme) y del dualismo sustancial de las primeras Méditations Métaphysiques. Por otro, Descartes siempre insistió en el hilemorfismo, composición, permixtio e incluso unión de las dos sustancias. Así, el cuerpo humano se hace depositario de propiedades peculiares tales como el ensamblaje, la indivisibilidad y el interés biológico en conservarse, lo que parece introducir cierta teleología en una parte de la extensión.
\end{abstract}

Palabras clave: Merleau-Ponty, Descartes, fenomenología, corporalidad, percepción, cogito, mecanicismo.

\section{SEE AND THINK. CARTESIAN MECHANISTIC PHYSIOLOGY AND PHENOMENOLOGY OF THE BODY}

As a tenacious and rigorous reader of the Cartesian corpus, Merleau-Ponty payed special attention to its ambiguities. On the one hand, the intellectualism of the Cartesian theory of perception (Dioptrique) goes along with a mechanistic physiology (Traite de l'Homme) and also with the substantial dualism of the first Méditations Métaphysiques. On the other, Descartes always insisted on hylomorphism, composition, permixtio and even substantial union. Thereby, the human body becomes endowed with such peculiar properties as its inner binding, indivisibility and a biological self-interest, instilling a certain teleology into a part of extension.

KEY WORDS: Merleau-Ponty, Descartes, phenomenology, corporeality, perception, mechanicism. 
$\overline{R M}$ En este trabajo nos propondremos ofrecer una visión sinóptica de las diversas interpretaciones que, a lo largo de la evolución de su obra, Merleau-Ponty desarrolló acerca de la filosofía de Descartes, poniendo especial atención en las cuestiones de la percepción y la corporalidad. Las reflexiones Merleau-Pontyanas ponen de manifiesto una ambigüedad en la obra cartesiana muchas veces no percibida en las lecturas clásicas del cartesianismo: la tensión entre el intelectualismo del que es subsidiaria la fisiología mecanicista, por un lado, y por otro, la antropología cartesiana basada en la noción de la unión psicofísica.

Para exponer sistemáticamente los diversos recodos interpretativos de los dichos y escritos de Merleau-Ponty acerca de Descartes dividiremos nuestro trabajo en dos partes. En la primera presentaremos la crítica al polo intelectualista de la filosofía cartesiana, tomando como ejemplos paradigmáticos las consideraciones disímiles de ambos autores en torno al fenómeno del miembro fantasma y la explicación de la percepción de la distancia. En la segunda parte nos centraremos en la elucidación del polo "unitarista", presentando el análisis Merleau-Pontyano del doblez implícito en el cogito y la explicitación de tal doblez en la noción cartesiana de la unión sustancial. Finalmente, con el fin de evaluar la interpretación Merleau-Pontyana que otorga un valor filosófico clave al concepto de la unión, introduciremos algunas hipótesis acerca de las modificaciones que se observan en la última obra de Descartes respecto de la caracterización de la corporalidad humana. Estas variaciones sugieren que la unión del cuerpo y el alma que caracteriza al ser del hombre implica la necesidad de una cierta revisión del marco mecanicista.

\section{Las criticas Merleau-Pontyanas al intelectualismo perceptivo y a la fisiología mecanicista}

Merleau-Ponty desarrolló su propia reflexión en diálogo constante con la filosofía cartesiana. Sus lecturas atentas de Descartes se reflejaron ya desde su primera obra publicada, La structure du comportement (1943), donde analiza largamente ciertos párrafos de la Dioptrique, hasta sus últimos cursos en el Collège de France: su muerte lo encontró en mayo de 1961 precisamente preparando un curso sobre Descartes, y leyendo aquella misma obra. Su pensamiento es generalmente caracterizado situándolo en la línea de las filosofías contemporáneas que identifican al cartesianismo con el dualismo, el idealismo y el intelectualismo, e intentan oponérsele y superarlo. De hecho así parece presentarse el mismo Merleau-Ponty frente al "gran público" en la primera de sus siete conferencias radiales de 1948. Refiriéndose a cierto "sentido común" que reserva a la ciencia el valor de "última palabra" respecto de lo que el mundo es y desvaloriza así las descripciones dóxicas de la experiencia vivida, Merleau-Ponty imputa a Descartes el habernos enseñado "a descubrir la impostura de [nuestros] sentidos y aprender a no confiar sino en la inteligencia" (Merleau-Ponty 2003, p. 10). El argumento cartesiano del pedazo de cera (Meditaciones metafísicas II) es presentado allí de modo simplista como un modelo del intelectualismo de la teoría cartesiana de la percepción. Si vemos 
el mismo trozo de cera a pesar de que sus presentaciones sensibles son diversas y cambiantes, ello se debería a que

"la verdadera cera no se ve con los ojos. Solo es posible concebirla con la inteligencia. Cuando yo creo ver la cera con mis ojos, lo único que hago es pensar, a través de las cualidades que caen por su propio peso, en la cera desnuda y sin cualidades que es su fuente común" (Merleau-Ponty 2003, pp. 11, 12).

En esta interpretación apresurada -que contrasta con las que Merleau-Ponty brindaría del pasaje en otros textos más estrictamente filosóficos-no solo la percepción sensible resultaría devaluada por Descartes como un mero "comienzo de ciencia todavía confusa" sino que necesitaría recurrir siempre al auspicio de la inteligencia para aprehender objetos idénticos y estables. Descartes habría dado pie a tales ideas al oponer abiertamente a la concepción aristotélico-escolástica de que "no hay nada en el entendimiento que antes no haya estado en los sentidos" su propia doctrina de que "ni nuestra imaginación ni nuestros sentidos podrían asegurarnos jamás de cosa alguna sin la intervención de nuestro entendimiento" (Descartes VI 1996, p. 37). De sentencias tales, Merleau-Ponty deriva en ocasiones una interpretación idealista de la teoría cartesiana de la percepción, que en su Fenomenología de la percepción identifica como el germen de la tradición moderna de las "filosofías trascendentales", tradición de la que participarían de distintos modos el kantismo y la fenomenología husserliana expuesta en Ideas $\mathrm{I}^{1}$.

En la visión de Merleau-Ponty, una teoría intelectualista de la percepción como la que habría esbozado Descartes sería necesariamente solidaria de una fisiología mecanicista como la que desarrolla el Tratado del hombre, es decir, de una concepción del cuerpo viviente como un ensamble de partes que se transmiten el movimiento lineal y causalmente tal como sucede con todas las partes del mundo físico. De acuerdo con esta primera visión estándar y simplificada de la relación de Merleau-Ponty con el cartesianismo que estamos reseñando, el filósofo habría comenzado por mostrar los límites de la fisiología mecanicista (en La structure du comportement y la Parte I de la Phénoménologie de la perception) para desarrollar a continuación (por ejemplo, en la Parte II de esta última obra) una filosofía no intelectualista de la percepción. Solamente si es posible mostrar que el cuerpo no es una mera "máquina tonta" sino que tiene sus modos particulares de conciencia e inteligencia se haría posible dispensar a la percepción de la necesidad de recurrir a representaciones, conceptos, categorías o juicios que sinteticen, organicen o den forma al flujo cambiante y múltiple de lo sensible. La structure $d u$

$1 \quad$ Esta fenomenología "estática” es diferenciada por Merleau-Ponty de las posteriores etapas "genética" y "generativa" de la fenomenología husserliana (propias de textos como las Meditaciones Cartesianas, la Krisis, Ideas II o los manuscritos de Husserl de la década de 1930 que Merleau-Ponty conocía directamente de los Archivos). En estas otras vertientes de la reflexión de Husserl, Merleau-Ponty inspiró en cambio gran parte de su propia reflexión. Así, afirma por ejemplo que "mientras la fenomenología no se haya convertido en fenomenología genética los retornos ofensivos del pensamiento causal y el naturalismo estarán justificados" (Merleau-Ponty 1957, p. 143). 
comportement intenta mostrar que la misma fisiología contemporánea experimental ha atravesado los límites de las explicaciones mecanicistas, aun si en ocasiones sigue intentando con dificultades expresar sus resultados en términos clásicos. Insertar el funcionamiento del cuerpo en el mundo físico demandaba explicarlo en términos de dependencias causales lineales entre estímulos simples, órganos receptores específicos, localizaciones cerebrales y eventualmente músculos efectores (Merleau-Ponty 1957, pp. 79 ss.). Sin embargo, según Merleau-Ponty, la experimentación reflexológica misma comprueba que:

a) un mismo estímulo es percibido de distintos modos y/o da lugar a distintas respuestas, dependiendo de su relación con otros estímulos anteriores o simultáneos, lo que implica que el cuerpo no capta estímulos simples sino más bien relaciones y diferencias entre estímulos (es decir, estructuras complejas o Gestalten).

b) los órganos sensoriales y motores del cuerpo -así como las neuronas- no están asignados al cumplimiento de una única función fija como las partes de una máquina sino que en ocasiones pueden alternarse, relevarse y cambiar de función de acuerdo a su relación con los demás en el contexto del organismo como totalidad funcional sinérgica o integrada.

c) la situación pragmática en la que el organismo se encuentra, así como la posición inicial de los órganos efectores determinan cuáles estímulos son captados y el modo en que lo son, dando lugar a respuestas diferentes. Esto implica un tipo de funcionamiento que no se atiene a la línea causal de estímulo y respuesta, circuitos nerviosos aferentes y eferentes, sensibilidad y motricidad. En la percepción y el comportamiento se establece entre el cuerpo y el mundo una suerte de "causalidad circular" que no podría llamarse propiamente "causalidad" sino más bien "sistema" o "estructura" (MerleauPonty 1975, p. 33).

Esta plasticidad y coordinación hacen pensar, más que en el modelo de una máquina circunscripta a leyes físicas, en un "cuerpo inteligente" que cuenta con cierta conciencia de sí mismo como totalidad integrada, de modo de servirse estratégicamente de sus recursos en vistas de realizar cierta acción con un sentido original y propiamente biológico. Evidentemente este tipo de "comprensión" o "conciencia corporal" no resulta de un proceso intelectual y consciente, pero tampoco según Merleau-Ponty puede explicársela exhaustivamente refiriéndola a una localización o a un proceso cerebral como el mapeo sensorio-motriz del propio cuerpo que los neurocientíficos contemporáneos localizan en los lóbulos frontal y parietal, el así denominado "homúnculo de Penfield" (Ramachandran 1999). Para sustentar este último punto, el filósofo se refiere a un caso de distorsión de la propiocepción al que también se había referido Descartes: el de la persistencia de sensaciones usualmente dolorosas percibidas en un miembro que ha sido amputado -"el miembro fantasma"-, cuestión a la que nos referiremos a continuación. 


\section{I.1. El caso del miembro fantasma}

La explicación cartesiana del fenómeno del miembro fantasma recurre tanto a una representación mental como a un acontecimiento nervioso-cerebral y/o solamente cerebral coordinado con la primera. Descartes sostiene, en primer lugar, que "si alguna causa no en el pie sino en otra cualquiera de las partes por las que se extienden los nervios del pie al cerebro, o aun en el propio cerebro, excita el movimiento que suele provocarse al herirse un pie, se sentirá el dolor como en el pie" (Descartes 1996 VII, p. 87; VIII, p. 319). Ahora bien, este evento fisiológico mecánico, comparable exactamente al tirar de una cuerda por un extremo o por su mitad produciendo en ambos casos el mismo movimiento en el otro extremo (el cerebro y, más exactamente, para Descartes, la hipófisis) sería simultáneo a la sensación de "dolor en el pie" que aparece en el alma por la sola institución divina, en beneficio del bienestar y la conservación del ser humano: "la naturaleza del hombre podría haber sido constituida por Dios de manera que el mismo movimiento en el cerebro mostrase alguna otra cosa al alma" (Descartes 1996 VII, p. 88).

La interpretación Merleau-Pontyana del fenómeno difiere de la cartesiana al menos en dos aspectos. En primer lugar, si bien la sensación de dolor es para Descartes una muestra contundente de la estrecha unión del alma con un cuerpo (y más precisamente, con una parte del cuerpo) que en este caso la afecta, no duda en considerar el dolor como un evento puramente mental. Así, en los Principios (Parte I, arts. XLVI y LXVII) el dolor es el ejemplo elegido para mostrar que una percepción puede ser clara aunque no sea distinta: el dolor es percibido vívida y claramente, pero se lo percibe como alojado en un parte del cuerpo en lugar de considerarlo distintamente tal como es, aconteciendo "en nuestra sola mente". Merleau-Ponty, en cambio, prefiere dar crédito a la experiencia del dolor físico tal como es vivida - es decir, de modo localizado- en vez de considerar esta experiencia de la localización dolorosa, tal como hace Descartes, como una derivación de "los prejuicios de la niñez". Así, encuentra en esta espacialidad o "voluminosidad del dolor" una indicación para pensar el cuerpo como sujeto de la percepción sensible y no solo como su causa exterior (Merleau-Ponty 1957, p. 100).

En lo que respecta al resto de la explicación cartesiana del miembro fantasma -la que compete al funcionamiento mecánico de los nervios y el cerebro- ésta también encuentra para Merleau-Ponty sus limitaciones. En primer lugar, la sensación del miembro fantasma puede subsistir aún anestesiando el muñón, es decir, en ausencia de estímulos periféricos. Esto no es un obstáculo definitivo para la explicación cartesiana ni para las contemporáneas explicaciones neurocientíficas del fenómeno, por cuanto ambas privilegian el rol del cerebro. Ahora bien, el situar en el cerebro, en una parte suya o en una red funcional neuronal la causa de la propiocepción comporta también sus problemas. En primer lugar, deja sin explicación el hecho de que el miembro fantasma no es sentido con la continuidad que tiene su presunto sustrato neuronal sino que aparece y desaparece intermitentemente, y en relación con situaciones, objetos o ambientes que tienen algún significado personal, pragmático o afectivo para el sujeto (Merleau-Ponty 1957, pp. 82, 83). En segundo lugar, se comprueba que el mapeo 
cerebral del propio cuerpo es plástico y se transforma paulatinamente, necesitando este cambio otras instancias explicativas que podrían no ser privativamente cerebrales ${ }^{2}$.

Razones como estas llevan a Merleau-Ponty a considerar que la clave irreductible de la propiocepción y el comportamiento sinérgico del cuerpo debe situarse en la totalidad del cuerpo mismo, pero ya no considerado en los términos objetivos de la anátomo-fisiología mecanicista sino en los de su vivencia subjetiva, i.e., como un sistema de disposiciones y capacidades de comportamiento y acción habituales en ciertos medios. Simultáneamente a la auto-organización del cuerpo en la acción, se da la organización de un mundo de objetos percibidos como campo donde tales acciones son posibles Si el cuerpo es considerado más allá de los límites del marco mecanicista puede prescindirse para Merleau-Ponty del recurso al intelecto para dar cuenta de la percepción de objetos, un recurso del que -de acuerdo a esta primera interpretación-no habría podido librarse la teoría cartesiana de la percepción.

\section{I.2. El caso de la percepción de la distancia}

Un caso paradigmático que muestra la diferencia entre una teoría intelectualista de la percepción como la que Merleau-Ponty atribuye en diversas ocasiones a Descartes y una teoría corporal y pragmática como la que esboza Merleau-Ponty es el de la percepción de la distancia y el tamaño de los objetos. En la explicación cartesiana de la Dióptrica la distancia es comparada con la altura de un triángulo cuya base (la distancia entre los dos ojos) y los ángulos de la base (la orientación de la mirada) me son conocidos. Descartes sostiene que esta captación consiste en un razonamiento geométrico o un juicio -es decir, una acción del pensamiento- y que éste no es percibido como tal por estar habituados a realizarlo desde la infancia, dando pie a una interpretación intelectualista de su teoría de la percepción: en definitiva, no es el ojo el que ve, sino el alma ${ }^{3}$. Este razonamiento dará por resultado una medida que podrá corresponderse con la distancia realmente existente -mediando la institución divina, como veremos- porque el mundo físico mismo, incluido en él el cuerpo, tiene una estructura matemática. Así es que deducir matemáticamente (intelectualmente) el tercer vértice de un triángulo para percibir la distancia puede ser comparado por Descartes con aprehender con los extremos de dos varas un objeto, formando físicamente un triángulo. Es por una "geometría natural" (Descartes) o, para usar un término de Malebranche que Merleau-Ponty asocia a las tesis de la Dioptrique, un “juicio natural” (Merleau-Ponty 1975, p. 274 ;1957, p. 284) -en

2 Estudios neurocientíficos experimentales recientes acerca de la plasticidad de la representación cortical somatosensorial (particularmente los emprendidos por V. S. Ramachandran y sus colegas) brindan explicaciones plausibles de los diversos modos en que ésta puede modificarse tras una amputación. A este respecto, cf. Gallagher 2005, pp. 98-99.

3 “(...) y nos hacen percibir la distancia del punto X: y esto, por una acción del pensamiento, que, no siendo sino una imaginación muy simple, no deja de implicar en sí un razonamiento semejante al que hacen los agrimensores cuando por medio de dos estaciones diferentes miden los lugares inaccesibles" (Descartes 1996 VI, p. 138). 
el sentido de un juicio no intelectual ni formulado sino de algún modo "cristalizado" en el mundo físico y realizado por el solo funcionamiento mecánico del cuerpo- que puede explicarse que los animales se comporten "como si percibieran" distancias. Puede decirse incluso que "perciben", como Descartes propone en la Regla XII o en el Tratado del hombre, con el recaudo de tomar este término en un sentido puramente fisiológico y mecánico sin la concomitancia mental que acaece en el ser humano, como mera transmisión causal del movimiento de partículas desde el exterior hasta el interior del cuerpo y en sentido inverso ${ }^{4}$.

Por otro lado, hay que recordar que Descartes hace de la percepción un juego de decodificaciones que permite pasar de los modos de la extensión a los modos del pensamiento o representaciones sin que haya allí ningún nexo efectivo sino solo la institución de un código por parte de Dios: se trata de lo que algunos intérpretes llaman una "teoría de la institución natural". Esta preordenación por parte de Dios de un sistema de correlaciones de acuerdo al cual a ciertos movimientos de la materia corresponden ciertas modificaciones del pensamiento sin que entre ambos se mantenga semejanza, establece un corte tajante entre lo extenso (incluido el propio cuerpo/ cerebro) y lo mental. Merleau-Ponty sugiere que lo que Descartes parece haber advertido correctamente en la Dióptrica (aun si hubiera resuelto erróneamente la dificultad) es que el ángulo de convergencia de los ojos o la triangulación de los dos bastones son solamente signos que deben ser interpretados para que simbolicen la profundidad espacial. Para Merleau-Ponty esta dotación de sentido a los signos físicos no podría realizarla un razonamiento geométrico que meramente replicaría la estructura formal que primeramente le prestó al mundo físico: “los 'signos' que, por hipótesis, tendrían que introducirnos en la experiencia del espacio, no pueden significar el espacio más que si ya están presos en él y si éste es ya conocido" (Merleau-Ponty 1957, p. 284). Sería necesario entonces suponer la prioridad tanto temporal como lógica de una experiencia perceptiva, corporal y pragmática del espacio como fuente originaria de sentido de la idealización geométrica, sin la cual esta última no hubiera siquiera tenido razón de desarrollarse. Al percibir la distancia de un objeto respecto de mi cuerpo estoy efectivamente tomándola como equivalente a la distancia que percibiría un observador situado en un tercer vértice formado por él, mi cuerpo y el objeto, es decir que estoy, aunque sea implícitamente, considerando la distancia como el ancho visto de perfil. De acuerdo con Merleau-Ponty, es por la posibilidad corporal de movernos y cambiar el punto de vista que conocemos estas equivalencias, y por la posibilidad indefinida de reiterar este cambio de perspectiva que se habría arribado a la concepción de un espacio isotrópico, homogéneo y sin orientación, tal como si fuera visto desde todas y desde ninguna perspectiva: el espacio geométrico ${ }^{5}$.

4 Cf. Reglas para la dirección del espiritu (Descartes 1996 X, p. 412 ss.); Tratado del hombre (Ibíd. XI, p. 131); Dióptrica (Ibíd. VI, p. 141 ss.).

5 Cf. a este respecto el capítulo III de la Parte I de la Fenomenología de la percepción: "La espacialidad del propio cuerpo y la motricidad". 
Pero si este espacio tiene utilidad y sentido para nosotros es porque significa al menos ciertos aspectos del espacio vivido corporalmente, en el que al movernos aprendimos el significado de estar arriba o abajo, delante o detrás, lejos o cerca: aprendimos en suma lo que el espacio es. La distancia percibida no es un valor que crece y decrece en un vector numérico homogéneo, sino que este vector espacial cuantitativo solo tiene sentido como abreviatura de algunos rasgos discretos de nuestra experiencia corporal en la que aprendimos pragmáticamente el sentido del espacio. En la espacialidad vivida las cosas no se nos acercan o alejan meramente como un punto respecto de otro, sino que se transforman en otras si están demasiado cerca, o se vuelven menos nítidas si están demasiado lejos. Oscilan en torno a un punto de equilibrio que está determinado por mis capacidades motrices (prioritaria pero no exclusivamente las de mi mirada en este caso) para hacer algo con ellas o "arreglármelas con ellas". De acuerdo con MerleauPonty, es de esta lejanía y cercanía "absolutas" y no mensurables (determinadas por una situación pragmática) que surge el sentido del espacio, y de ellas toman prestado su sentido las lejanías y cercanías relativas de un cuerpo respecto de otro en el espacio físico o geométrico. Descartes habría intuido que el espacio percibido contiene un plus de sentido respecto del espacio físico tal como él lo entendía y por ello éste necesitaba de una interpretación para hacerse equivalente al primero, pero este suplemento, según Merleau-Ponty, no podría proveerlo un razonamiento que captara su estructura geométrica sino la experiencia del comportamiento corporal.

\section{La ambigüedad cartesiana: Descartes más allá de la interpretación intelectualista}

$\mathrm{Si}$, tal como vimos, Merleau-Ponty intenta desarrollar una crítica de concepciones intelectualistas de la percepción y mecanicistas del cuerpo, y en repetidas ocasiones ve en el cartesianismo a un exponente de ambas, se muestra también sumamente consciente a lo largo de la evolución de toda su obra de que el pensar de Descartes no puede ser reducido a tales términos. Desde su primera obra hasta sus últimos cursos son frecuentes en Merleau-Ponty las referencias a una ambigüedad, ambivalencia, "estrabismo" o "diplopía" cartesianas. El intelectualismo y el mecanicismo cartesianos serían solamente una cara de su filosofía derivada de una de las dos interpretaciones a las que se presta su cogito, ambas interpretaciones abiertas incluso, según MerleauPonty, por Descartes mismo. Según La estructura del comportamiento, la duda metódica lleva a Descartes a descubrir un cogito que está "muy cerca de la noción moderna de la conciencia" y su inseparable correlato, "el dominio indubitable de las significaciones" (Merleau-Ponty 1975, p. 272). En esta dimensión caracterizada en los términos vagamente "fenomenológicos" de una conciencia no calificada, no tendría sentido alguno la distinción entre el entendimiento y la percepción sensible, así como entre el cuerpo y el alma. Según Merleau-Ponty no podría hablarse en este punto con propiedad de un sujeto meramente intelectual de la percepción en Descartes, ya que no hay términos exteriores a esta experiencia que permitan caracterizarla como privativamente mental (distinguiéndola de otro ámbito) y, por consiguiente, la percepción 
no podría "aparecer ya como el efecto en nosotros [en nuestra mente] de la acción de una cosa exterior, ni el cuerpo como intermediario de esa acción causal" (Merleau-Ponty 1975, p. 272). Las nociones de una "composición" e incluso una "unión sustancial” de mente y cuerpo reflejarían posteriormente esta intuición cartesiana básica de un ámbito "fenomenológico" no calificado e indistintamente mental y corporal, intuición original que las formulaciones dualistas habrían traicionado.

Esta "traición" a la evidencia fenomenológica de una conciencia indiferente a la distinción sustancial se comprobaría ya en el ejemplo del pedazo de cera, que en La estructura del comportamiento Merleau-Ponty considera con más detalle que en las conferencias más arriba citadas. Considerando el pasaje en el contexto total de las Meditaciones metafísicas, puede deducirse que el análisis intelectual y el juicio al que alude el pasaje nos dan solamente la esencia de la cosa, "la estructura inteligible de los objetos percibidos" (Merleau-Ponty 1975, p. 272), mientras que, como dirá la Meditación VI, la imaginación nos da su objeto como "presente" y la percepción nos lo presenta sin haberlo querido, aportando un índice existencial que manifiesta "alguna cosa que difiere de mi espíritu” (Descartes 1996 VII, p. 73). Desde esta perspectiva, mi cuerpo y el mundo serán objetos reales distintos de mí, la percepción deberá ser una representación mental al término de una cadena causal que pasa por el cuerpo y comienza en el estímulo físico, y la conciencia deberá ser definida finalmente como puramente inmaterial. Desde estas premisas no habría integración real entre la intelección y la sensación: "No es en el alma, es en Dios, donde se enlazan la una a la otra" (ídem). En efecto, tanto en la Dióptrica como en las Reglas y hasta en los Principios se establece que las cosas físicas no se relacionan con las percepciones sensibles que de ellas tenemos por semejanza -así como tampoco las palabras se asemejan a las representaciones que suscitan en nosotros- sino por una "institución de la naturaleza" que es propiamente el arbitrario decreto divino que sella su relación. Esta desemejanza se comprobaría claramente en el caso de lo que posteriormente la tradición filosófica moderna denominaría "cualidades secundarias", tales como los colores, olores y sabores, o en el de la sensación de dolor a la que ya aludimos más arriba: no habría nada en el mundo semejante a tales sensaciones sino tan solo figuras, formas, movimientos. Pero incluso la percepción de las propiedades primarias o matematizables como la distancia -a la que ya nos referimos también- recurriría a la arbitraria institución divina, que en este caso particular decretaría que las propiedades percibidas se correspondan con propiedades semejantes de las cosas realmente existentes, mientras que podrían no hacerlo. Así, Descartes afirmaba ya en la Dióptrica (VI) que el alma conoce directamente la situación de los objetos sin deducir la posición de los miembros (y la correlativa localización del objeto) de la imagen cerebral que de ella se imprime, sino que atiende solamente a la disposición de las partes del cerebro y ve directamente la situación de los objetos a causa de la mentada institución de la naturaleza (cf. Merleau-Ponty 1975, p. 274, 266).

Para salvar esta escisión aparece en los textos cartesianos la noción de "unión" del alma con el cuerpo, un concepto que resultaría ininteligible mientras el alma siguiera concibiéndose como siendo en esencia un pensamiento inmaterial y el cuerpo como una máquina automática. En este sentido interpreta Merleau-Ponty en su Fenomenología la correspondencia entre Descartes y la princesa Elizabeth, referida específicamente a 
la cuestión. Según indica en esta obra Merleau-Ponty, de las respuestas epistolares que brinda Descartes se concluiría que la unión es una noción primitiva e innata que ha sido puesta por Dios en nosotros y no es posible otorgar acerca de ella mayores explicaciones: "detrás del hombre como es de hecho se encuentra Dios como autor razonable de nuestra situación de hecho. Apoyado en esta garantía trascendente, Descartes puede aceptar tranquilamente nuestra situación irracional" (Merleau-Ponty 1957, p. 215). En sus cursos de 1947-48 en la École Normale, así como en sus cursos de 1956 en el Collège de France acerca de la concepción cartesiana de la naturaleza, Merleau-Ponty se refiere a otra alternativa que ensayó ocasionalmente Descartes (Merleau-Ponty 1995, pp. 2537). O bien, como en la correspondencia con Elizabeth de Bohemia, se cierra la puerta a mayores indagaciones metafísicas acerca de la composición de estos incomposibles, remitiendo la comprensión de la unión al Creador que instituyó en nosotros su noción primitiva, o bien se redefinen los términos del compuesto para hacerlos compatibles. Siguiendo esta segunda línea, Descartes se habría referido circunstancialmente a la posibilidad de pensar el espíritu como corporal (cartas a Arnauld y Morus ${ }^{6}$ ) o a la posibilidad de dotar al cuerpo humano de propiedades que, estrictamente según sus propios términos, solamente podrían corresponder al alma, tales como la indivisibilidad (Tratado de las pasiones del alma I, XXX).

La estructura del comportamiento intenta hacer sentido de estas ambigüedades, otorgando crédito filosófico a ambas vertientes de la reflexión cartesiana-la de la unión y la del dualismo-, e incluso admitiendo una relativa justificación fenomenológica para la opción dualista. Según aquella obra, la dimensión fenomenológica de la experiencia tal como es vivida es "un medio neutro respecto a las distinciones substanciales entre el organismo, el pensamiento y la extensión"; en ella "el ego (...), el cuerpo y las cosas (...) no están confundidos, pero no son más que tres sectores de un campo único" (MerleauPonty 1975, p. 264). Sin embargo, en ciertas situaciones, tales como la enfermedad, la conciencia descubre "una resistencia del cuerpo propio. Puesto que una herida en los ojos basta para suprimir la visión, es que vemos entonces a través del cuerpo". Tales circunstancias proveerían un fundamento fenomenológico para el pensamiento dualista, según el cual "el cuerpo [como una cosa más] hace de pantalla entre nosotros [definidos ahora como conciencias inmateriales] y las cosas" (Merleau-Ponty 1975, p. 264). Según el filósofo, "en estos casos de desintegración, el alma y el cuerpo son aparentemente distintos, y ésta es la verdad del dualismo" (Merleau Ponty 1975, p. 290). Sin embargo, se trata aquí, según Merleau-Ponty, de una verdad secundaria y derivada de la más primitiva unión que se comprueba en la experiencia usual. Cuerpo y alma deberían ser vistos más bien como "nociones relativas", dimensiones de una misma estructura: "no es una dualidad de sustancias" (Merleau-Ponty 1975, p. 291).

Este es un punto particularmente interesante de la lectura Merleau-Pontyana de los textos de Descartes, ya que la lectura de Merleau-Ponty parece hacer lugar a

$6 \quad$ Citadas por M. Merleau-Ponty en sus cursos de la École Normale Supériere (1947-1948) sobre L'union de l'âme et du corps chez Malebranche, Biran et Bergson (1978, p. 14). 
dos concepciones que muchos exégetas cartesianos consideran en la actualidad como mutuamente excluyentes ${ }^{7}$.

\section{II.1. La primacía del cogito vertical}

El último curso dictado por Merleau-Ponty e interrumpido por su muerte se refiere justamente a Descartes, y en él el filósofo se propone ahondar en la génesis de la ambivalencia cartesiana, así como en el doblez que atraviesa en su visión al cogito. En este curso, Merleau-Ponty pone atención en ciertas metáforas y términos recurrentes de Descartes, tales como la "luz natural", la "visión del espíritu" o lo "puro", no para considerarlos como claves de una explicación psicologista de una filosofía sino como "cifras de una ontología" (Merleau-Ponty 1996, p. 228). En las Reglas (III), Descartes concibe la intuitus mentis tomando expresamente como modelo la visión ocular, y más precisamente según Merleau-Ponty, un modelo sesgado y deficiente de la visión. Para Descartes, la visión del espíritu, tal como la de los ojos, se dirige a singula puncta, detalles o figuras presentes. El resto, lo no visto, está puramente ausente o es inexistente. Desde un modelo fenomenológico de la visión como el que adopta Merleau-Ponty, en cambio, nada puede ser visto más que en su relación con un "horizonte" como el que conforman las perspectivas ocultas del objeto o el fondo sobre el que se destaca su figura. Esta relación con lo no visto determina que lo que se ve sea visto del modo en que lo es, de modo que lo no visto tiene un modo particular de visibilidad, al que los fenomenólogos aluden precisamente con la noción de "horizonte": aun si no está presente como lo que ocupa el foco de atención, el horizonte visual no es una pura ausencia. Incluso el "campo visual" no es un cuadro con límites definidos, sino que se funde con sus horizontes: "Hay ahí una visión indeterminada, una visión de no se sabe qué, y en el límite, lo que está detrás de la espalda no carece de presencia visual" (Merleau-Ponty 1957, p. 5). En la visión focal y aislante sobre la que Descartes modela el pensamiento no hay espacio para este carácter "horizóntico" de latencia, difuminación e indeterminación que es propio de la visión tal como se da: se ve o no se ve. De ahí que, según Merleau-Ponty, la distinción -y no la claridad- es la posibilidad de la visión

7 Algunos intérpretes, tales como M. Wilson (1990) o S. Voss ("Descartes: The End of Anthropology", en Cottingham 1994) consideran que la teoría de la institución natural es suficiente para dar cuenta de todos los cuestionamientos que podrían hacerse al dualismo del sistema cartesiano y que, por lo tanto, la noción de "unión” y las explicaciones ligadas a ella que Descartes presenta, por ejemplo, en la correspondencia con Elizabeth, en las réplicas a Gassendi y en la correspondencia con Regius, serían completamente innecesarias. Por otro lado, hay quienes consideran que la respuesta cartesiana última al problema de la comunicación entre mente y cuerpo se presenta mediante la noción primitiva de "unión", la cual sería irreductible a las también primitivas nociones de mente y cuerpo. Esta noción de unión sería la que permitiría explicar un fenómeno como el de la percepción sensible, en el cual se hallan implicados tanto modos de la extensión como modos del pensamiento. M. Rozemond (1998), J. Cottingham (1985) o R. Richardson (1982) serían ejemplos de este segundo tipo de lectura. 
intelectual que más aprecia Descartes: la luz natural es una luz concentrada en lugar de difusa (Merleau-Ponty 1996, p. 334) y la intuitus mentis "comporta una exclusión de todo lo demás que la visión de los ojos no comporta nunca más que relativamente"; hay aquí "un todo o nada de intelección” (Merleau-Ponty 1996, pp. 230, 231).

Ahora bien, "el tipo de interrogación que se introduce con las dos primeras Meditaciones representa una puesta en cuestión de (...) [la] restricción inicial [cartesiana] del Ser al Ser de las figuras" (ibíd., p. 239). La duda deja al pensamiento o a la visión intelectual "en suspenso". Bajo la sombra de la duda ya no se sabe si se ve o si no se ve, si algo es o no es, y, sin embargo, bajo esta luz ya no focal sino difusa que se atisba entre el ser y la nada para Descartes sigue habiendo algo, lo que desafía su modelo de intelección previo. Se da en este punto, según Merleau-Ponty, un "cambio de sentido de la luz natural" que antes era propiamente visión distinta (Merleau-Ponty 1996, p. 257). Entre lo que se ve y lo que se niega, o más bien conteniendo a ambos, aparece el "yo soy", "pero no como ser totalmente positivo, en sí, (...) sino como aliquid, diferente de nada, como ese al que todo se aparece" (ídem): aquello que Descartes refiere como el "tesoro de mi espíritu" y Merleau-Ponty como la apertura de "un campo de experiencias" (Merleau-Ponty 1957, pp. 445, 446). Algo aparece precisamente cuando la luz natural "renuncia a ser inmediatamente verdad (distinción)", y aparece precisamente "porque acoge todo (soñar, sentir, imaginar) sin rechazar nada en nombre de la distinción" (Merleau-Ponty 1996, p. 267). La evidencia del cogito en su sentido prístino no es entonces para Merleau-Ponty una evidencia por distinción, sino justamente por indistinción o mélange (mezcla).

Eso que aparece en la penumbra -cuando por un instante, en la Meditación II, no se pretende aún definir qué es sino solo constatar que es-y que Descartes llama Ego, no es primeramente una sustancia inteligida, no es la idea de duda o de cogitatio: para Merleau-Ponty no se trata aquí todavía de un conocimiento por idea. En los términos del filósofo, "el intelecto del que llego a formar la idea secundariamente es el ser representativo u objetivo de un intelecto operante que yo soy, y éste (...) no se puede concebir como una luz sin ninguna sombra" (Merleau-Ponty 1996, p. 254). MerleauPonty diferencia así entre "un cogito operante" o "vertical" y un "cogito reflexivo", "enunciado" u "horizontal". El primero funda al segundo, y no puede caracterizarse por la simplicidad de una naturaleza pura (ídem). El cogito en su significado originario (vertical) no es entonces para Merleau-Ponty una construcción sustancialista sino el simple "estar abierto a" un campo de experiencias, sean éstas de la índole que sean: "La cogitatio es lo que comprendo de mí, y (...) no es falso, pero (...) es derivado de [una] constatación o experiencia" previa (Merleau-Ponty 1996, p. 249).

En la interpretación Merleau-Pontyana, si el yo es comprendido en su realidad formal y no objetiva, primariamente como existencia de la que después se extraerán las ideas de cogitatio, intellectus, animus o mens, no puede afirmarse que el Yo del cogito sea entendimiento puro. El cogito vertical expresa indistintamente "un contacto presignificativo con este pensamiento naturante que soy, y con este cuerpo mío cuya unión conmigo es per se notum" (Merleau-Ponty 1996, p. 254). Es porque el cogito operante o vertical, no aún objetivado y definido, reúne en su indistinción el alma y el cuerpo, que puede reunir también para Descartes el inteligir, el afirmar, el querer, el imaginar 
y el sentir, antes de que lo asocie más estrecha y privativamente con el entendimiento y entonces deba considerar que el ver es propio del cogito solo como "pensamiento de ver". Según Merleau-Ponty, en el argumento del pedazo de cera puede observarse este ambiguo juego cartesiano entre un cogito vertical y operante que es propiamente visión a mismo título que intelección y un cogito horizontal que es "pensamiento de ver" y no visión a secas. Esta ambigüedad podría resumirse como "todo es Cogitatio, pero la Cogitatio es todo": la primera fórmula apuntaría a un intelectualismo perceptivo, mientras que la segunda sugeriría que el cogito, en tanto es también percepción sensible, no puede definirse como puramente intelectual. Cuando Descartes afirma que yo veo por la mirada del espíritu la cera que creía ver con los ojos parece agregar al proceso perceptivo una función intelectual distinta de la sensible, pero para que yo siga viendo siempre la misma cera y el pensamiento pueda pasar por visión, debería pensarse más bien en una conciencia indeterminada, intelectual y sensible a la vez. La intuición primordial de Descartes - la del cogito vertical- debería ser entendida entonces como la de una experiencia que aúna en su indistinción y su riqueza pensamiento y cuerpo, entendimiento y sensibilidad (Merleau-Ponty 1996, p. 266).

\section{II.2. La noción primitiva de "unión” y los intereses del cuerpo}

Es posible hallar en Descartes mismo razones para avalar la interpretación de MerleauPonty según la cual la unión de alma y cuerpo debe considerarse portadora de un valor propiamente filosófico más allá de su mera constatación o validez pragmática. Por una parte puede confirmarse que, tal como observaba Merleau-Ponty, en aquellos pasajes de su correspondencia en los que Descartes parece sugerir que el pensamiento podría ser dotado de cierta extensión, rápidamente señala que tal concepción carece de rigor científico y no es más que una constatación de nuestras vivencias cotidianas. Dentro de este grupo de aseveraciones cabe citar el pasaje de la carta a Morus en la que se atribuye al alma una "extensión de potencia" por la cual se explicaría su interacción con el cuerpo (Descartes 1996 V, p. 342). Tal vez el pasaje de la obra cartesiana más contundente en este sentido sea la afirmación hecha en una de las primeras cartas intercambiadas con Elizabeth de Bohemia en la que Descartes recomienda, para comprender el modo en que alma y cuerpo se conectan, "atribuir libremente materia y extensión al alma, pues esto no es otra cosa que concebirla unida al cuerpo" ${ }^{8}$. Pero también en este caso el texto cartesiano continúa indicando que una vez concebida de este modo la unión, resultará más sencillo entender intelectualmente la distinción real entre la mente y el cuerpo.

Por otra parte, sin embargo, existen otros pasajes en los que se presenta la unión psicofísica no ya como un dato de la vida cotidiana carente de valor filosófico o científico sino como un punto de partida para llevar a cabo un análisis en physicien-como físico

$8 \quad$ Cf. carta a Elizabeth del 28 de junio de 1643 (Descartes 1996 III, p. 693 ss.), la carta a Regius de enero de 1642 (ídem, p. 492), la respuesta a las objeciones hechas por Arnauld (ibíd. VII, p. 227) y la carta a Mesland de febrero de 1645 (ibíd. IV, p. 167). 
o como filósofo natural- de las pasiones que afectan al alma en su unión con el cuerpo. Tal es el caso del Tratado de las pasiones del alma, cuyo primer artículo señala:

"Y, para empezar, considero que todo lo nuevo que se hace o que sucede generalmente es denominado por los filósofos una pasión respecto al sujeto al que sucede y una acción respecto a aquel que hace que suceda. De suerte que, aunque el agente y el paciente sean a menudo muy diferentes, la acción y la pasión no dejan de ser siempre una misma cosa" (Descartes 1996 XI, p. 328; el destacado es nuestro).

Este suceso único que posee dos caras (pasión y acción) solo permite ser comprendido a partir de la noción de "unión" presente ya desde la Sexta Meditación, mediante la cual se bosqueja la antropología cartesiana como una dimensión distinta a la metafísica. La primera aparición de la noción de la "unión" de la mente y el cuerpo en el hombre se da en la comparación recién aludida con el piloto y su barco, presente tanto en la quinta parte del Discurso del método como en la sexta de las Meditaciones metafísicas. Recordemos esta última:

"La naturaleza me enseña también por medio de estos sentimientos de dolor, de hambre, de sed, etc., que yo no estoy alojado en mi cuerpo como un piloto en su navío, sino que estoy muy estrechamente unido y confundido y mezclado de tal modo que formo como un único todo con él" (Descartes 1996 VII, p. 81).

Un primer punto a tener en cuenta aquí es que es en la sensación y no en la causación de la mente sobre el cuerpo (acción voluntaria) donde se pone de manifiesto que ambas sustancias forman en el hombre un todo unificado. Descartes infiere a partir de la existencia de modos de pensamiento oscuros y confusos (sensaciones y sentimientos) que un hombre no es una mente que interactúa causalmente con un cuerpo sino una entidad más compleja en la que se "entremezclan" el cuerpo y el alma. Un segundo punto a considerar es que Descartes no está presentando una explicación acerca de cómo es que alma y cuerpo actúan el uno sobre el otro, sino caracterizando el tipo de conexión que hay entre ellos. En la carta a Regius de enero de 1642 se afirma algo muy similar a lo recién referido, pero esta vez mediante la comparación entre el modo en que se encuentran unidos el cuerpo y el alma de un hombre y la conexión meramente ocasional que se daría en el caso de un entendimiento puro que "utilizara" un cuerpo:

"Percibimos que los sentimientos de dolor y todos los de naturaleza similar no son puros pensamientos del alma en cuanto distinta del cuerpo, sino confusas percepciones de este alma que está realmente unida al cuerpo, pues si un ángel estuviera unido al cuerpo humano no tendría sentimientos como los nuestros, sino que solamente percibiría los movimientos causados por los objetos externos, distinguiéndose por tal razón de un verdadero hombre" (Descartes 1996 III, pp. 492, 493).

De ambos textos podemos concluir que la mente del hombre cartesiano no está aparte de su cuerpo sino que se encuentra "encarnada" en él, y esta afirmación se funda en un dato de la experiencia: la sensación es una forma particular de percepción que está presente en el hombre pero que, según Descartes, no podría darse en el caso 
de sustancias pensantes que no conformaran una unión de materia y pensamiento, y donde se establecieran entre ambas sustancias solo relaciones causales (si tales cosas existieran). Sin embargo, hay que observar que en ninguno de los textos la unión es invocada como explicación del modo en que la interacción se produce, ni tampoco como condición necesaria para el movimiento del cuerpo: se afirma, en cambio, que es condición necesaria para que el alma pueda tener sensaciones y sentimientos. Tal como subrayaba Merleau-Ponty, Descartes encuentra todo esto en el ámbito de las enseñanzas de la naturaleza, diferenciado claramente del dominio de la luz natural.

Tal como observábamos más arriba, es en la última obra cartesiana publicada -a la que Merleau-Ponty no dedicó análisis tan exhaustivos- donde es posible hallar claves para evaluar la interpretación Merleau-Pontyana de Descartes en la que la unión psicofísica acarrea una significación filosófica de peso. En el Tratado de las pasiones del alma encontramos una serie de elementos que rompen con la consideración meramente mecanicista del cuerpo, al mismo tiempo que desdibujan la distinción entre las enseñanzas de la naturaleza y la luz natural ${ }^{9}$. Entra en juego aquí, por ejemplo, el interés del sujeto como totalidad psicofísica, y en función de este interés surge una dimensión normativa de acuerdo con la cual el sujeto -entendido ahora como alma encarnada o cuerpo animado- valora prejudicativamente sus experiencias como buenas o malas para sí mismo. Tanto las percepciones que el sujeto refiere (rapport) a su propio cuerpo (dolor, hambre, sed, etc.) como las que refiere al alma (amor, deseo, tristeza, etc.) poseen una utilidad para la conservación del compuesto psicofísico, en la medida en que proveen de significación a la escena en la que el sujeto se encuentra, de acuerdo al beneficio o la nocividad que comporta para él lo que lo rodea. El cuerpo, entonces, en la medida en que integra una unión psicofísica no puede ser descripto adecuadamente en términos meramente maquínicos, pues surge para él un fin del que carece la extensión por sí misma: el de conservarse siendo lo que es, lo cual implica también el conservarse unido a esa alma ${ }^{10}$. Esta reintroducción de las causas finales se hace manifiesta, por ejemplo, en el artículo 52, cuando se señala que "la utilidad de todas las pasiones consiste tan solo en que predisponen al alma para que quiera las cosas que nos son útiles, según dicta la naturaleza, y para que persista en esta volición" (Descartes 1996 XI, p. 372).

El cuerpo unido realmente a un alma, a diferencia de cualquier otro trozo de extensión, contiene en sí una intención o interés por conservarse siendo lo que es. Este interés, al mismo tiempo que lo "organiza" y le da identidad, provee al cuerpo de una

$9 \quad$ En la clasificación de pensamientos que se presenta entre los artículos 17 y 25 del Tratado se analizan en pie de igualdad las ideas claras y distintas y las oscuras y confusas, en la medida en que todas ellas son percepciones recibidas pasivamente por el alma.

10 Hay que recordar que entre las novedades que Descartes introduce respecto de la fisiología escolástica está el hecho de considerar a la muerte no como un efecto de la separación entre el cuerpo y el alma que la informa, sino como la causa de tal separación. La organización del cuerpo se pierde y es por ello que el alma ya no puede mantenerse unida a él. Cf. a este respecto el artículo VI de la Parte I del Tratado de las pasiones del alma. 
unidad interna. El cuerpo deja así de ser una totalidad de partes extra partes para ser una totalidad orgánica, tal como se señala en el artículo 30, cuyo título es "El alma está unida a todas las partes del cuerpo conjuntamente". Allí Descartes afirma que:

"Es preciso saber que el alma está realmente unida a todo el cuerpo y que no se puede decir con propiedad que esté en alguna de sus partes con exclusión de las otras, porque él es uno, y de alguna manera indivisible, en razón de la disposición de sus órganos, los cuales se relacionan de tal modo el uno con el otro que, cuando se suprime alguno de ellos, todo el cuerpo se torna defectuoso; y porque ella es de una naturaleza que no tiene relación con la extensión, ni con las dimensiones u otras propiedades de la materia de las que el cuerpo se compone, sino solo con la ensambladura toda de sus órganos" (Descartes 1996 XI, p. 351).

En estos párrafos del Tratado de las pasiones del alma puede advertirse cómo la noción de unión o compuesto sustancial, que constituye uno de los polos en tensión que MerleauPonty reconoce en la obra cartesiana, trae aparejada un cambio considerable respecto de la premisa mecanicista de que toda explicación del movimiento de los cuerpos puede agotarse en la explicitación de una cadena de causas eficientes. Pero además, se introduce aquí una dimensión en la que hasta entonces Descartes prácticamente no había reparado: se trata de la dimensión temporal en la que el hombre como compuesto tiene que moverse. Desde el punto de vista del entendimiento puro, la temporalidad es solo una marca de la finitud del sujeto, en la medida en que el descubrimiento de las ideas claras y distintas que se encuentran en él de manera innata solo puede hacerse en el transcurso temporal. Pero una mente desencarnada no tiene urgencia, no le va la vida en ello. En el Tratado de las pasiones del alma, en cambio, nos encontramos con que la temporalidad en la que se sitúa el sujeto es la temporalidad del obrar, y no ya la del conocimiento de las verdades eternas ${ }^{11}$. Si se vuelve necesaria la pasión como una suerte de conocimiento abreviado, aunque incierto, respecto de lo conveniente y lo nocivo es porque este sujeto se encuentra obligado a obrar y no posee, en tanto es un cuerpo, más que un breve instante para determinar su acción. Pero nuevamente, esta consideración de la temporalidad se liga a un ámbito de intereses que carece absolutamente de sentido en relación con la mera aglomeración de partes que constituye una máquina. Una máquina no tiene la pretensión ni la intención de perdurar, ni tampoco puede adaptar o modificar la secuencia de movimientos que le han sido impresos por su constructor en vistas de tal propósito, por lo cual si existen las pasiones es solo porque un cuerpo humano, en esta consideración tardía de Descartes, es algo cuyo obrar no puede explicarse de modo puramente mecánico ${ }^{12}$.

11 Estrictamente, una reflexión semejante podría también desarrollarse a partir de lo expresado por Descartes en la Sexta Meditación, donde se plantean las ideas confusas de la sensación como enseñanzas de la naturaleza que guían la acción de un sujeto que posee un cuerpo y carece de la ciencia completa para determinar su voluntad. Cf. Descartes 1996 VII, p. 83.

12 En el artículo 137 se define el uso natural de la pasión como "el de incitar al alma a consentir y contribuir a las acciones que pueden servir para conservar el cuerpo, o para hacerle de alguna manera más perfecto" (Descartes 1996 XI, p. 430). 


\section{Consideraciones finales}

En las sucesivas lecturas que desarrollara de la obra de Descartes, Merleau-Ponty se mostró sensible a las complejidades de la reflexión cartesiana acerca de la percepción y la corporalidad. Ciertamente, a la hora de definir por contraste su propia teoría, Merleau-Ponty adoptó con frecuencia una interpretación llanamente intelectualista de la gnoseología cartesiana, según la cual la percepción sensible consistiría básicamente en la yuxtaposición de una fisiología mecánica y el suplemento de una actividad intelectual añadida en el caso del ser humano. Para sortear los problemas de una teoría de esta índole -solo superados por Descartes mediante el recurso a una institución divina- sería necesario, desde la visión Merleau-Pontyana, abandonar el modelo del cuerpo-máquina y de la causalidad eficiente y lineal entre partes, por el de un cuerpo que se auto-organiza y adquiere una suerte de conciencia unitaria de sí mismo en virtud de sus solas disposiciones comportamentales. Una reformulación tal de la noción del cuerpo llevaría aparejada simultáneamente y por necesidad una nueva definición de la conciencia como no privativa ni esencialmente intelectual, sino también y a mismo título sensible y corporal. Merleau-Ponty ve reflejada una intuición semejante en la experiencia originaria de un cogito "vertical" - caracterizado como indistinto, operante, irreflexivo y no enunciado- del que derivaría secundariamente el cogito sustancial que habría dado pie a las formulaciones dualistas: aquel que según Descartes no es propiamente “visión" sino solo "pensamiento de ver". Desde este marco interpretativo, la noción cartesiana de la unión psicofísica adquiere un sentido y un valor no solo pragmático sino filosófico y pasa incluso a ocupar el primer plano. En ciertos textos de Descartes referidos a sensaciones y sentimientos, pudimos constatar que el filósofo advirtió la necesidad filosófica de caracterizar la relación entre el alma y el cuerpo más allá de la mera interacción causal. La última obra del filósofo muestra incluso que el cuerpo humano mismo en tanto está unido a un alma adquiere propiedades distintas de las de cualquier máquina, tales como la indivisibilidad y la "ensambladura" integral de sus partes en vistas de un interés propio, caracteres que se acercan a las mismas nociones que Merleau-Ponty opondría luego al intelectualismo cartesiano.

\section{Referencias bibliográficas}

Cottingham, J. (1985), “Cartesian Trialism”, Mind 94: 218-230. Press. (ed.) (1994), Reason, Will and Sensation. Oxford: Oxford University

Descartes, R. (1996), Oeuvres de Descartes (ed. Adam-Tannery). París: Vrin.

Gallagher, Shaun (2005), How the Body Shapes the Mind. New York: Oxford University Press.

Guéroult, M. (1953), Descartes selon l'ordre des raisons (2 vols.). París: Aubier.

Merleau-Ponty, M. (1978), L'union de l'âme et du corps chez Malebranche, Biran et Bergson (1947-1948). París: Vrin. 
(1957), Fenomenología de la percepción (trad. esp. de E. Uranga). México: Fondo de Cultura Económica.

(1975), La estructura del comportamiento (trad. esp. de E. Alonso). Buenos Aires: Hachette.

(1995), La Nature. Notes. Cours du Collège de France. París: Éditions du Seuil.

(1996), Notes des Cours au Collège de France (1958-1959 et 19601961). París: Gallimard.

(2003), El mundo de la percepción. Siete conferencias. Buenos Aires: Fondo de Cultura Económica.

Ramachandran, V. S. (1999), Fantasmas en el cerebro. Madrid: Editorial Debate.

Richardson, R. (1982), “The 'Scandal' of Cartesian Interactionism”, Mind. XCI: pp. 20-37.

Rozemond, M. (1998), Descartes's Dualism. Cambridge: Harvard University Press.

Wilson, M. (1990), Descartes. México: UNAM. 